\title{
The Bretón-Manko equatorially antisymmetric binary configuration revisited
}

\author{
V. S. Manko ${ }^{\dagger}$, R. I. Rabadán ${ }^{\dagger}$ and E. Ruiz ${ }^{\ddagger}$ \\ ${ }^{\dagger}$ Departamento de Física, Centro de Investigación y de Estudios Avanzados del IPN, A.P. 14-740, 07000 México D.F., Mexico \\ ${ }^{\ddagger}$ Instituto Universitario de Física Fundamental y Matemáticas, \\ Universidad de Salamanca, 37008 Salamanca, Spain
}

\begin{abstract}
The Bretón-Manko solution for two identical counter-rotating Kerr-Newman charged masses is rewritten in the physical parametrization involving Komar quantities. The new form of the solution turns out to be very convenient for verifying that the black-hole sector of the Bretón-Manko binary configuration saturates a recent geometric inequality for interacting black holes with struts discovered by Gabach Clement.
\end{abstract}

PACS numbers: 04.20.Jb, 04.70.Bw, 97.60.Lf

\section{INTRODUCTION}

The Bretón-Manko (BM) stationary axisymmetric electrovac solution of the Einstein-Maxwell equations [1] was constructed in 1995 with the aid of Sibgatullin's integral method [2, 3]. It is asymptotically flat and describes a system of two identical counter-rotating Kerr-Newman (KN) sources, black holes or hyperextreme objects. The sources in the BM configuration are prevented from falling onto each other by a massless strut [4], the latter representing a specific line source of pressure, which is removable only in the special case of equal masses and charges when the solution becomes a specialization of the Parker-Ruffini-Wilkins hyperexteme field [5] generated by means of the Perjés-IsraelWilson method $[6,7]$. The BM solution gave rise to a systematic study of equatorially antisymmetric spacetimes [8-10] which were shown to constitute a rather broad and interesting subfamily of stationary axisymmetric fields. At the same time, the BM solution has also enlarged a class of metrics able to describe the interacting black holes - up to date the particular members of that class have been obtained and analyzed mainly in the context of the static vacuum [11-13], electrostatic [12, 14-17] or stationary vacuum [18-20] spacetimes with struts. While the paper [1] basically deals with the mathematical structure of the BM metric and associated equilibrium problem, it would certainly be of interest to better explore the physical properties of the spacetime whose subextreme sector provides excellent opportunities for the study of two interacting KN black holes and for extending the recent results on interacting Kerr sources [18] to the case of non-vanishing electromagnetic field. It would be also desirable to use the general BM metric for testing the newly proposed geometric inequality [21] for multiple black holes with struts.

To make the BM solution more suitable for the physical analysis, in the present paper we shall rewrite it in a new parametrization involving the physical Komar quantities [22] and a separation distance as arbitrary parameters. This will enable us to demonstrate analytically that each KN black-hole constituent from the subextreme sector of the BM solution verifies the well-known Smarr's mass formula [23]. We will subsequently apply the BM solution for demonstrating that it saturates the inequality on the bounds of the interaction force in black-hole systems with struts recently derived by Gabach Clement [21].

\section{THE BM SOLUTION IN PHYSICAL PARAMETERS}

The original BM solution arises from the axis data

$$
\begin{aligned}
& \mathcal{E}(\rho=0, z)=\frac{\left(z-k-M-i a^{\prime}\right)\left(z+k-M+i a^{\prime}\right)}{\left(z-k+M-i a^{\prime}\right)\left(z+k+M+i a^{\prime}\right)}, \\
& \Phi(\rho=0, z)=\frac{2 Q z}{\left(z-k+M-i a^{\prime}\right)\left(z+k+M+i a^{\prime}\right)},
\end{aligned}
$$

representing the axis expressions of the Ernst complex potentials [24]. The parameters $M, a^{\prime}, Q$ and $k$ in (1) are related, respectively, to the mass, angular momentum, charge of each KN source and to the separation distance, $\rho$ and $z$ are the Weyl-Papapetrou cylindrical coordinates. In the paper [1], Sibgatullin's method was applied to the data (1) for obtaining the corresponding Ernst potentials in the whole space, as well as the metric functions $f, \gamma$ and $\omega$ entering the line element

$$
d s^{2}=f^{-1}\left[e^{2 \gamma}\left(d \rho^{2}+d z^{2}\right)+\rho^{2} d \varphi^{2}\right]-f(d t-\omega d \varphi)^{2} .
$$

The explicit original form of $\mathcal{E}, \Phi, f, \gamma$ and $\omega$ of the BM solution the reader may find in [1]. 
It should be pointed out that the parametrization used in [1] is not the most attractive one from the physical point of view because only the parameters $M$ and $Q$ coincide exactly with the physical mass and charge of each KN constituent; at the same time, the parameter $a^{\prime}$ does not coincide with the angular momentum per unit mass of any of the constituents, whereas the parameter $k$ is not exactly half the coordinate distance between the centers of the constituents. In the absence of the electric charge $Q$ (pure vacuum case) when the BM solution represents two counter-rotating Kerr sources [25] a similar problem was already solved in the paper [18] by introducing the Komar angular momentum per unit mass $a$ instead of $a^{\prime}$, and the coordinate distance $R$ between the centers of the sources instead of $k$ (see Fig. 1).

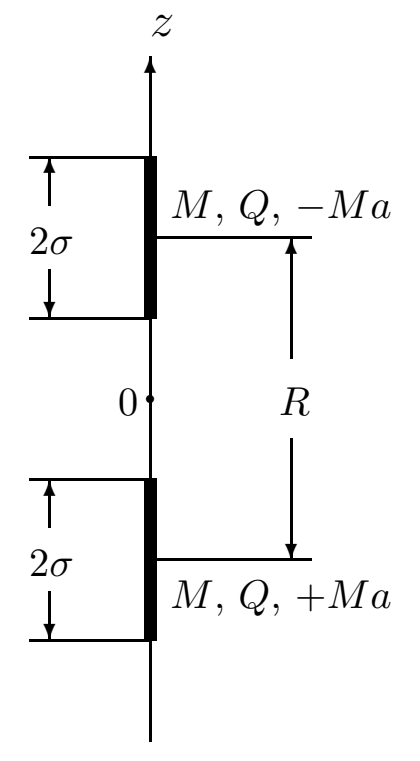

FIG. 1: Location of identical counter-rotating KN black holes on the symmetry axis and the new parametrization.

Remarkably, transition from the parameters $\left(a^{\prime}, k\right)$ to the physically more transparent parameters $(a, R)$ can be performed in the general BM solution too, and after a considerable effort we have eventually been able to find the required reparametrized form of the data (1) and the form of an important quantity $\sigma$ generalizing the analogous quantity in the vacuum case (cf. formula (3) of [18]):

$$
\begin{aligned}
& \mathcal{E}(\rho=0, z)=\frac{z^{2}-2 M z-\left(\frac{1}{2} M R+M^{2}-\frac{1}{2} Q^{2}-i M a\right)^{2} \mu}{z^{2}+2 M z-\left(\frac{1}{2} M R+M^{2}-\frac{1}{2} Q^{2}-i M a\right)^{2} \mu}, \\
& \Phi(\rho=0, z)=\frac{2 Q z}{z^{2}+2 M z-\left(\frac{1}{2} M R+M^{2}-\frac{1}{2} Q^{2}-i M a\right)^{2} \mu},
\end{aligned}
$$

and

$$
\sigma=\sqrt{M^{2}-Q^{2}-M^{2} a^{2} \mu}, \quad \mu:=\frac{R^{2}-4 M^{2}+4 Q^{2}}{\left(M R+2 M^{2}-Q^{2}\right)^{2}} .
$$

The new parameters $R$ and $a$ are related to $a^{\prime}, k, M$ and $Q$ by the formulae

$$
\begin{aligned}
R & =2 \kappa_{+}, \quad a=\frac{a^{\prime} k\left(2 M^{2}-Q^{2}+2 M \kappa_{+}\right)}{M\left(M^{2}-k^{2}+a^{\prime 2}+4 Q^{2}-d\right)}, \\
\kappa_{+} & =\sqrt{\left(k^{2}+M^{2}-a^{\prime 2}-2 Q^{2}+d\right) / 2}, \quad d=\sqrt{\left(k^{2}-M^{2}+a^{\prime 2}\right)^{2}+4 M^{2} a^{\prime 2}} .
\end{aligned}
$$

The application of Sibgatullin's method to the data (3) yields the following new expressions for the Ernst potentials of the BM solution instead of the old ones:

$$
\mathcal{E}=\frac{A-B}{A+B}, \quad \Phi=\frac{C}{A+B},
$$




$$
\begin{aligned}
A= & \left(M^{2}-Q^{2}\right)\left[4 \sigma^{2}\left(R_{+} R_{-}+r_{+} r_{-}\right)+R^{2}\left(R_{+} r_{+}+R_{-} r_{-}\right)\right]+\left[\sigma^{2}\left(R^{2}-4 M^{2}+4 Q^{2}\right)\right. \\
& \left.-M^{2} a^{2} R^{2} \mu\right]\left(R_{+} r_{-}+R_{-} r_{+}\right)-2 i a M R \mu \sigma\left(M R+2 M^{2}-Q^{2}\right)\left(R_{+} r_{-}-R_{-} r_{+}\right), \\
B= & 2 M R \sigma\left\{R \sigma\left(R_{+}+R_{-}+r_{+}+r_{-}\right)-\beta\left(R_{+}-R_{-}-r_{+}+r_{-}\right)\right\}, \\
C= & Q B / M, \\
R_{ \pm}= & \sqrt{\rho^{2}+\left(z+\frac{1}{2} R \pm \sigma\right)^{2}}, \quad r_{ \pm}=\sqrt{\rho^{2}+\left(z-\frac{1}{2} R \pm \sigma\right)^{2}}, \quad \beta:=2\left(M^{2}-Q^{2}\right)+i M a \mu\left(M R+2 M^{2}-Q^{2}\right) .
\end{aligned}
$$

On the other hand, the new expressions for the functions $f, \gamma$ and $\omega$ of the BM solution have been found to have the form

$$
\begin{aligned}
f= & \frac{A A^{*}-B B^{*}+C C^{*}}{(A+B)\left(A^{*}+B^{*}\right)}, e^{2 \gamma}=\frac{A A^{*}-B B^{*}+C C^{*}}{16 R^{4} \sigma^{4} R_{+} R_{-} r_{+} r_{-}}, \omega=-\frac{\operatorname{Im}\left[2 G\left(A^{*}+B^{*}\right)+C I^{*}\right]}{A A^{*}-B B^{*}+C C^{*}}, \\
G= & -z B+R \sigma\left\{\left(2 M^{2}-Q^{2}\right)\left[2 \sigma\left(r_{+} r_{-}-R_{+} R_{-}\right)+R\left(R_{-} r_{-}-R_{+} r_{+}\right)\right]\right. \\
& \left.+M(R+2 \sigma)(R \sigma-\beta)\left(R_{+}-r_{-}\right)+M(R-2 \sigma)(R \sigma+\beta)\left(R_{-}-r_{+}\right)\right\}, \\
I= & \frac{Q}{M}\left\{G+R Q^{2} \sigma\left[2 \sigma\left(r_{+} r_{-}-R_{+} R_{-}\right)+R\left(R_{-} r_{-}-R_{+} r_{+}\right)\right]\right\},
\end{aligned}
$$

where an asterisk denotes complex conjugation and $\operatorname{Im}(x)$ the imaginary part of $x$. Notice that the metric function $\omega$ in (7) is presented in a simpler and more elegant form than in the paper [1].

For completeness, below we also write out expressions for the potentials $A_{4}$ and $A_{3}$ of the BM solution which represent, respectively, the electric and magnetic components of the electromagnetic 4-potential $A_{i}=\left(0,0, A_{3}, A_{4}\right)$ :

$$
A_{4}=\operatorname{Re}\left(\frac{C}{A+B}\right), \quad A_{3}=\operatorname{Im}\left(\frac{I}{A+B}\right)
$$

where $\operatorname{Re}(x)$ means taking real part of $x$. The knowledge of the above $A_{4}$ and $A_{3}$ is needed in particular for the analysis of Smarr's mass formula.

\section{SOME PHYSICAL PROPERTIES OF BM SOLUTION}

The real valued $\sigma$ determine a black-hole sector of the BM solution. In this case there are two horizons ${ }^{1}$ determined by the null hypersurfaces $\rho=0, \frac{1}{2} R-\sigma \leq z \leq \frac{1}{2} R+\sigma$ and $\rho=0,-\frac{1}{2} R-\sigma \leq z \leq-\frac{1}{2} R+\sigma$, which in the cylindrical coordinates $(\rho, z)$ are represented by rods of length $2 \sigma$ (see Fig. 1). Each KN constituent then must verify Smarr's mass formula for black holes

$$
M=\frac{1}{4 \pi} \kappa S+2 \Omega^{H} J+\Phi^{H} Q
$$

which, apart from the mass $M$, angular momentum $J$ and charge $Q$, contains four quantities to be evaluated on the horizon: the surface gravity $\kappa$, the area of the horizon $S$, horizon's angular velocity $\Omega^{H}$ and the electric scalar $\Phi^{H}$. Since the constituents in the BM solution only differ in the orientation of their angular momenta, it is sufficient to check formula (9) for solely one subextreme constituent, say, the upper one; the second constituent will then have opposite angular momentum $-J$ and horizon's velocity $-\Omega^{H}$ whose product leaves invariant the second term on the right hand side of (9). The known Komar characteristics associated with the upper constituent are $M, J=-M a, Q$, and this fact can be checked straightforwardly by using Tomimatsu's formulae [29]

$$
\begin{aligned}
M & =-\frac{1}{8 \pi} \int_{H} \omega \Omega_{, z} \mathrm{~d} \varphi \mathrm{d} z \\
J & =\frac{1}{8 \pi} \int_{H} \omega\left[-1-\frac{1}{2} \omega \Omega_{, z}+\tilde{A}_{3} A_{3, z}^{\prime}+\left(A_{3} A_{3}^{\prime}\right)_{, z}\right] \mathrm{d} \varphi \mathrm{d} z \\
Q & =\frac{1}{4 \pi} \int_{H} \omega A_{3, z}^{\prime} \mathrm{d} \varphi \mathrm{d} z
\end{aligned}
$$

\footnotetext{
${ }^{1}$ They are in fact Killing horizons by the same argument as used in [26] for the case of the double-Kerr solution [27] (see p. 348 of [26]).
} 
where $\Omega=\operatorname{Im}(\mathcal{E}), A_{3}^{\prime}=\operatorname{Im}(\Phi), \tilde{A}_{3}=A_{3}-\omega A_{4}$, and the integration must be performed over the horizon $H$ on which the metric function $\omega$ and the potential $\tilde{A}_{3}$ take constant values. Obviously, the total mass, total angular momentum and total charge of the BM system are equal, respectively, to $2 M, 0$ and $2 Q$.

On the other hand, the quantities $\kappa, S, \Omega^{H}$ and $\Phi^{H}$ can be calculated with the aid of the formulae [28, 29]

$$
\kappa=\sqrt{-\omega^{-2} e^{-2 \gamma}}, \quad S=\frac{4 \pi \sigma}{\kappa}, \quad \Omega^{H}=\omega^{-1}, \quad \Phi^{H}=A_{4}-\Omega^{H} A_{3},
$$

thus yielding in our case

$$
\begin{aligned}
\kappa & =\frac{R \sigma}{\Delta}, \quad S=\frac{4 \pi \Delta}{R}, \quad \Omega^{H}=-\frac{M a \mu\left(M R+2 M^{2}-Q^{2}\right)}{\Delta}, \\
\Phi^{H} & =\frac{Q\left[(R+2 M)(M+\sigma)-2 Q^{2}\right]}{\Delta} \\
\Delta & =2 M(R+2 M)(M+\sigma)-Q^{2}(R+4 M+2 \sigma) .
\end{aligned}
$$

A direct check shows that the above quantities satisfy identically the relation (9), therefore the subextreme constituents in the BM solution are indeed charged rotating black holes.

Mention that in the limit $R \rightarrow \infty$ (infinite separation) one recovers from (12) the characteristics of a single KN black hole [30], and at finite $R$, formulae (12) take into account the interaction between the black holes. The interaction force in the BM configuration is determined by a very simple expression $\left(\gamma_{0}\right.$ is the value of the metric function $\gamma$ on the strut)

$$
\mathcal{F}=\frac{1}{4}\left(e^{-\gamma_{0}}-1\right)=\frac{M^{2}-Q^{2}}{R^{2}-4 M^{2}+4 Q^{2}},
$$

which coincides with the expression for the interaction force between two identical Reissner-Nordström non-rotating charged masses [31, 32] (cf. formula (30) of [15]). Mention that geometrically $\mathcal{F}$ can be defined in terms of the conical deficit $\delta$ associated with the strut as $\mathcal{F}=-\delta /(8 \pi)$. From (13) and (4) one easily infers that no equilibrium states of two identical counter-rotating black holes with non-zero angular momenta are possible without a supporting strut; at the same time, when $M^{2}=Q^{2}$, we have either equilibrium of two hyperextreme KN constituents $(a \neq 0)$, or that of two Reissner-Nordström extreme black holes $(a=0)$.

The limiting case of two extreme counter-rotating black holes is defined by the condition $\sigma=0$. Solving the latter for $R$, we find that $M$ and $Q$ must satisfy the inequality $M^{2}>Q^{2}$ as a condition of the reality of $R$ and non-vanishing $a$. On the other hand, solving $\sigma=0$ for $a$, we get

$$
a^{2}=\frac{M^{2}-Q^{2}}{M^{2} \mu}
$$

and taking into account that $0<M^{2} \mu<1$ for all $M>0, R>2 \sqrt{M^{2}-Q^{2}}$, we conclude that a black hole in the BM configuration needs a larger absolute value of the angular momentum in order to become extremal than a single KN black hole. The Ernst potentials and metric functions of the extreme BM solution have been worked out in explicit form in the paper [33].

Let us also comment, in connection with recent interest in geometrical inequalities for black holes (we refer the reader to a comprehensive review [34] on the topic), that the extreme BM solution provides the first example of a stationary axisymmetric electrovac spacetime for which the equality is achieved in a recent relation for black holes with struts derived by Gabach Clement [21]:

$$
\sqrt{1+4 \mathcal{F}} \geq \frac{\sqrt{(8 \pi J)^{2}+\left(4 \pi Q^{2}\right)^{2}}}{S}
$$

Indeed, taking into account (14), the extremal values of $S$ and $(8 \pi J)^{2}+\left(4 \pi Q^{2}\right)^{2}$ are

$$
\begin{aligned}
& S=\frac{4 \pi}{R}\left[\left(M^{2}-Q^{2}\right)(R+4 M)+M^{2} R\right], \\
& (8 \pi J)^{2}+\left(4 \pi Q^{2}\right)^{2}=\frac{16 \pi^{2}\left[\left(M^{2}-Q^{2}\right)(R+4 M)+M^{2} R\right]^{2}}{R^{2}-4 M^{2}+4 Q^{2}},
\end{aligned}
$$

with which, and with formula (13), the equality in (15) can be easily verified. In general, the inequality (15) establishes lower bounds on the interaction force between the black-hole constituents in the BM solution. 
We conclude this section by noticing that Smarr's formula gives rise to the inequality

$$
M \geq 2 \Omega^{H} J+\Phi^{H} Q
$$

since the first term on the right hand side of (9) is a non-negative quantity. Then the equality in (17) will be achieved exclusively by the extremal black holes, while the sub-extreme black holes will verify a strict inequality.

\section{CONCLUSIONS}

The new representation of BM solution elaborated in the present paper is remarkable in several aspects: it is the first and most simple example of a stationary axisymmetric electrovac spacetime entirely written in physical parameters and able to describe the field of two interacting KN black holes (previous results on the physical parametrization of binary configurations were obtained either for a vacuum [18] or static electrovac [15] cases). It considerably simplifies the analysis of physical properties of the BM solution and permits one to get concise expressions for the basic characteristics of interacting KN black holes and use them in concrete applications, in particular, for proving that the BM binary configuration saturates the Gabach-Clement inequality for interacting black holes with struts one of the main results of the present paper. The new form of the BM metric also motivates the search for physical parametrizations of more general configurations of $\mathrm{KN}$ black holes than the $\mathrm{BM}$ one, for instance by introducing appropriately the charges into the vacuum solution for non-equal counter-rotating Kerr black holes [35]. Last but not least, an interesting aspect of the reparametrized BM solution is connected with a recent numerical study by Dain and Ortiz [36] of the non-stationary axisymmetric binary black-hole systems using a procedure that employs stationary axisymmetric spacetimes with struts as "momentary stationary data", thus suggesting that the BM solution could in principle be used in the modern numerical codes for obtaining new information about the non-radiating binary non-stationary systems too.

\section{Acknowledgements}

We are grateful to the anonymous referee for many valuable comments and suggestions that helped us to considerably improve the presentation. We also thank Erasmo Gómez for technical computer support during the involved analytic calculations. This work was partially supported by CONACYT, Mexico, and by Ministerio de Ciencia y Tecnología, Spain, under the Project FIS2009-07238.

[1] Bretón N and Manko V S 1995 Class. Quantum Grav. 121969

[2] Sibgatullin N R 1991 Oscillations and Waves in Strong Gravitational and Electromagnetic Fields (Berlin: Springer)

[3] Manko V S and Sibgatullin N R 1993 Class. Quantum Grav. 101383

[4] Israel W 1977 Phys. Rev. D 15935

[5] Parker L, Ruffini R and Wilkins D 1973 Phys. Rev. D 72874

[6] Perjés Z 1971 Phys. Rev. Lett. 271668

[7] Israel W and Wilson G A 1972 J. Math. Phys. 13865

[8] Ernst F J, Manko V S and Ruiz E 2006 Class. Quantum Grav. 234945

[9] Ernst F J, Manko V S and Ruiz E 2007 Class. Quantum Grav. 242193

[10] Sod-Hoffs J and Rodchenko E D 2007 Class. Quantum Grav. 244617

[11] Israel W and Khan K A 1964 Nuovo Chim. 33331

[12] Costa M S and Perry M J 2000 Nucl. Phys. B 591469

[13] Emparan R and Reall H S 2002 Phys. Rev. D 65084025

[14] Emparan R and Teo E 2001 Nucl. Phys. B 610190

[15] Manko V S 2007 Phys. Rev. D 76124032

[16] Chng B, Mann R, Radu E and Stelea C 2008 J. High Energy Phys. 12009

[17] Stelea C, Dariescu C and Dariescu M 2011 Phys. Rev. D 84044009

[18] Manko V S, Rodchenko E D, Ruiz E and Sadovnikov B I 2008 Phys. Rev. D 78124014

[19] Herdeiro C A R and Rebelo C 2008 J. High Energy Phys. 10017

[20] Herdeiro C A R, Radu E and Rebelo C 2010 Phys. Rev. D 81104031

[21] Gabach Clement M E 2012 Class. Quantum Grav. 29165008

[22] Komar A 1959 Phys. Rev. 113934

[23] Smarr L 1973 Phys. Rev. Lett. 3071

[24] Ernst F J 1968 Phys. Rev. 1681415 
[25] Kerr R P 1963 Phys. Rev. Lett. 11237

[26] Dietz W and Hoenselaers C 1985 Ann. Phys. (NY) 165319

[27] Kramer D and Neugebauer G 1980 Phys. Lett. A 75259

[28] Carter B 1979 in General Relativity, an Einstein Centenary Survey, ed. S. W. Hawking and W. Israel (Cambridge University Press, Cambridge), p. 294.

[29] Tomimatsu A 1984 Prog. Theor. Phys. 7273

[30] Newman E T, Couch E, Chinnapared K, Exton A, Prakash A and Torrence R 1965 J. Math. Phys. 6918

[31] Reissner H 1916 Ann. Physik 50106

[32] Nordström G 1918 Proc. Kon. Ned. Akad. Wet. 201238

[33] Manko V S, Ruiz E and Sadovnikova M B 2011 Phys. Rev. D 84064005

[34] Dain S 2012 Class. Quantum Grav. 29073001

[35] Cabrera-Munguia I, Lämmerzahl C and Macías A 2013 Preprint gr-qc/1302.4843

[36] Dain S and Ortiz O E 2009 Phys. Rev. D 80024045 